\title{
Simulation Models of Energy Cables in SPICE
}

\author{
Uma Balaji \\ Department of Electrical and Computer Systems Engineering, Fairfield University, United States
}

\begin{tabular}{l} 
Article Info \\
\hline Article history: \\
Received Jul 24, 2017 \\
Revised Feb 23, 2018 \\
Accepted Mar 18, 2018 \\
\hline Keyword: \\
Electromagnetic interference \\
High frequency cable behavior \\
Spice cable model \\
Transient analysis \\
Transmission line
\end{tabular}

\begin{abstract}
Accurate modeling of cables is important to study the behavior of high frequency disturbances in power converter systems. This paper reviews and compares two popular methodologies to model energy cables - an improved per unit length parameters based model and a Laplace SPICE element based model. The two models presented take into account the frequency dependence of the parameters of the cable. A ladder network is used for this purpose in the per unit length parameters based model. The Laplace SPICE element model is generated from a rational function approximation for the admittance parameters that are frequency dependent. The rational function approximation is obtained using a well known vector fitting algorithm. The time and frequency domain solutions of a two wire energy cable, obtained from the two models, agree well.
\end{abstract}

Copyright (ㅇ 2018 Institute of Advanced Engineering and Science. All rights reserved.

Corresponding Author:

Uma Balaji,

Department of Electrical and Computer Systems Engineering,

Fairfield University,

1073, North Benson Road, Fairfield, CT- 06444

Email: ubalaji@fairfield.edu

\section{INTRODUCTION}

Power electronic systems in industrial applications often use long cables. Semiconductor devices used in such systems generate pulses with short rise and fall times and thus give rise to high frequency components in the currents and voltages [1]. The high frequency common mode and differential mode currents in cables produce emissions that can cause unwanted electromagnetic interference (EMI) [2]. Therefore, an accurate model of the cables that describes their high frequency behavior is necessary to compute the currents flowing in them and thereby predict any emissions produced. The power electronic semiconductor devices for drives or other applications are often modelled in SPICE [3]. The high frequency behavioral models of motors with the models of the power electronic system and cables are simulated in SPICE to predict the electromagnetic interference [4]. Hence, a high frequency circuit model of cable in SPICE is necessary. The signals flowing on long cables of power electronic systems are pulse width modulated (PWM). Such signals can be used as excitations in SPICE to test the cable model when the cable is terminated in any load.

Many existing models are available on standard SPICE simulators for two wire cables. One model based on transmission line equations simulates a lossless uniform transmission line. This model describes the cable by its characteristic impedance and signal propagation delay. Since this model does not include the cable losses, it is not suitable for use in applications to predict EMI when long cables are used. Another simple SPICE model is based on cascaded sections of pi, T or L-shaped equivalent circuits of per unit length R, L, G and C parameters distributed along the cable. The RLGC model ignores the variation of the per unit length resistance and inductance with frequency due to the skin effect and proximity effect. Similarly, the variation of the permittivity and loss tangent of the dielectric, which results in increased loss at higher frequencies, is not accounted for in this model. By ignoring those parametric variations, inaccurate time and frequency domain solutions are obtained from simulations. Several methods have been proposed to correct 
this by using improved models for the cable. Such models account for the variation of the parameters with frequency. One method has been the use of a ladder network to model the frequency dependence of losses [5]. Another method has been to use a rational function approximation of the admittance matrix of the cable [6-12]. These two methods have been presented and compared in this paper.

\section{THEORY}

Accurate analysis of EMI in power converter systems for drives requires high frequency circuit models of all the components in the system. A commercial tool, ANSYS-Simplorer that integrates multiple modelling technologies has been used to simulate the power electronic converter system [13]. Further this work used, finite element analysis based ANSYS-Maxwell to obtain the high frequency behavioral model of induction motor and Matlab-Simulink to simulate its control and study faults caused by different drive's components. The high frequency behavioral models of all the components in the power electronic systems including the cables can also be obtained from different software platforms and simulated in SPICE to predict EMI [4]. For this approach, two methods to obtain a SPICE cable model is reviewed and presented below.

The electrical properties of a transmission line are characterized by the per unit length parameters $\mathrm{R}$, $\mathrm{L}, \mathrm{G}$ and $\mathrm{C}$. The circuit theory-based definitions of the parameters are related to the stored electric and magnetic energies, as well as the power loss and obtained from a field theory-based analysis of the transmission line [14]. The transmission lines parameters of some common lines such as coaxial, two wire and parallel plate are determined at low frequency using analytical techniques. The lumped parameter SPICE model of the transmission line is obtained by using a number of L-shaped sections of the RLGC parameters distributed along the length of the cable. If $\mathrm{n}$ sections (L-shaped) are used per unit length as shown in Figure. 1, the lumped element values in each section are $\mathrm{R} / \mathrm{n}, \mathrm{L} / \mathrm{n} . \mathrm{G} / \mathrm{n}$ and $\mathrm{C} / \mathrm{n}$. The first and last series branches are half sections with lumped elements $R /(2 n)$ and $L /(2 n)$ to keep the line symmetric. There should be enough sections in the model to ensure that each section represents the length of the line that is a small fraction of the wavelength $(\lambda / 20)$ at the highest frequency of the signal flowing on the line. Depending on the rise time of the time domain signal, the highest frequency component on the line is estimated as 035/(rise time). Some designers use the estimate of $1 /$ (rise time) for the highest frequency component. In practice, by choosing the number of sections to be large enough in the model, the system behavior with a long cable is predicted in the time and frequency domains. However, this model is still insufficient to describe the effects of cable length on signal flow, as the per unit length parameters are frequency dependent. In order to determine the parameter values at high frequencies, numerical techniques, such as the finite element method, are used [15]. Their variations with frequency are later accounted in the SPICE model. One method to account for the frequency dependence of the parameters is the use of a ladder network in the model [5]. The losses due to skin effect, causing the cable behavior to be frequency dependent, are modelled by the RL ladder network. This ladder network is used in place of a single $\mathrm{R}$ and $\mathrm{L}$ in the series branch of Figure 1. The frequency dependent variation of the real and imaginary components of permittivity of the dielectric material of the cable is modelled with an RC ladder network. This ladder network is used in place of a single $\mathrm{G}$ and $\mathrm{C}$ in the shunt branch of Figure 1.

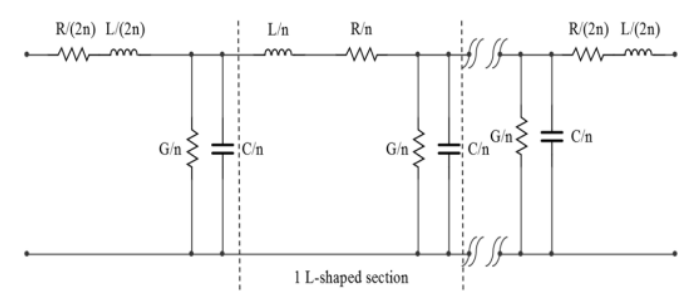

Figure 1. Transmission Line Model with $\mathrm{n}$ Sections of Per Unit Length Parameters R, L, G and C

Distributed on Unit Length of the Cable

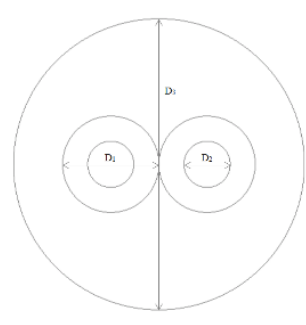

Figure 2. Cross-section of the Two Wire Cable:

Diameter of the Wires $\mathrm{D}_{2}=1.38 \mathrm{~mm}$, Diameter of the PVC Covering of Wires $\mathrm{D}_{1}=2.88 \mathrm{~mm}$, Diameter of the Rubber Shield Over the two Wires $\mathrm{D}_{3}=8.7 \mathrm{~mm}$, Relative Permittivity of

Rubber $=2.3$, Conductivity of the Wires $=45.94 \mathrm{MS} / \mathrm{m}$ 
Another method to account for frequency dependence of per unit length parameters is by directly using frequency dependent elements in the SPICE model. To do this, Laplace SPICE elements are used to represent the frequency dependent admittance parameter matrix of the long cable. A vector fitting algorithm approximates the admittance parameter matrix of the cable as a function of frequency [9] with a rational function in the Laplace domain. A Laplace SPICE element is then used to represent the rational function approximation. A model that characterizes the transmission line with the frequency dependent effects is thus obtained. The results from the ladder network SPICE model and Laplace element SPICE model are compared here. The ladder network model for a two wire cable, as presented in [5], has been calculated here for the purpose of comparing the two methods.

\subsection{Ladder Network Model for a Two Wire Cable}

The cross section of a $1 \mathrm{~m}$ two wire unshielded energy cable is shown in Figure 2. Using a finite element method-based electromagnetic solver tool (ANSYS-HFSS), the RLGC parameters of this cable were obtained over the frequency range $10 \mathrm{KHz}-100 \mathrm{MHz}$. These parameters can be also determined with a vector network analyzer. The $1 \mathrm{~m}$ long two wire cable was modelled with number of sections, $\mathrm{n}=32$. The time domain pulse with a rise time of $10 \mathrm{~ns}$ was used to test the model (highest frequency component of $100 \mathrm{MHz}$ ). With 32 section per meter of cable, the length of each section was much lower than $\lambda / 20$ at $100 \mathrm{MHz}$. The variations of resistance and inductance with frequency (series branch of Figure 1) are represented by an RL ladder network shown in Figure 3. A least square curve fitting algorithm was used to determine the elements of the ladder network. This algorithm minimized the difference in equivalent impedances of the ladder network and the series branch of the cable in the frequency range. The variations of conductance and capacitance parameters (shunt branch of Figure 1) with frequency are represented by the RC ladder network shown in Figure 4. Using the same least square curve fitting algorithm, the difference in admittances of the ladder network and the shunt branch were minimized. A SPICE sub circuits netlist for Figure 3 and Figure 4 are written with the determined ladder network element values. The SPICE model for the cable is created by including 32 sections of the sub circuits in the netlist.

The model has been validated by comparing the input impedances of the $1 \mathrm{~m}$-long cable terminated with either a short or open circuit. The experimental results for these configurations from [5] have been compared with the model obtained in this work as shown in Figure 5. Although the recalculated values of the elements of the ladder network vary slightly from the original work, the simulated impedances are in good agreement with the measurements.

The time domain response of the cable to a $40 \mathrm{~V}$ pulse with a rise and fall time of 10ns is shown in Figure 6. The applied input $40 \mathrm{~V}$ pulse was identical to the pulse in [5] and the transient response simulation from this work agrees well with measurements [5]. This solution is further compared with the model obtained from the admittance parameter matrix of the cable.

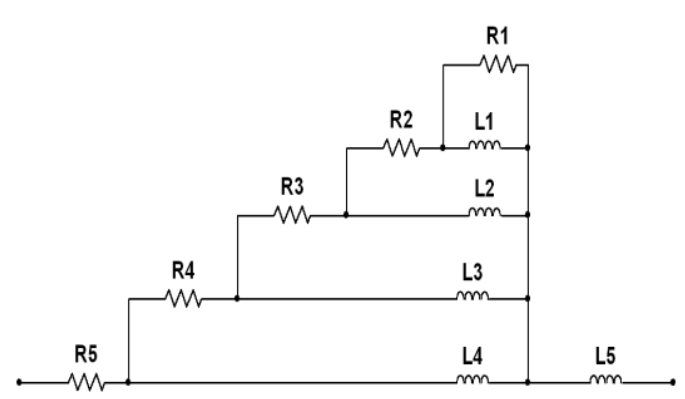

Figure 3. RL ladder network. For the $1 \mathrm{~m}$ cable of Figure. 2 with 32 sections, $\mathrm{R} 1=1.5 \mathrm{e}-1 \Omega$, $\mathrm{R} 2=9.9021 \mathrm{e}-2 \Omega, \mathrm{R} 3=7.5125 \mathrm{e}-2 \Omega, \mathrm{R} 4=4.25 \mathrm{e}-2 \Omega$, $\mathrm{R} 5=6.9246 \mathrm{e}-3 \Omega, \mathrm{L} 1=5.0002 \mathrm{e}-13 \mathrm{H}, \mathrm{L} 2=6.0733 \mathrm{e}-$ $11 \mathrm{H}, \mathrm{L} 3=6.0733 \mathrm{e}-11 \mathrm{H}, \mathrm{L} 4=3.4392 \mathrm{e}-10 \mathrm{H}$, $\mathrm{L} 5=1.7158 \mathrm{e}-08 \mathrm{H}$.

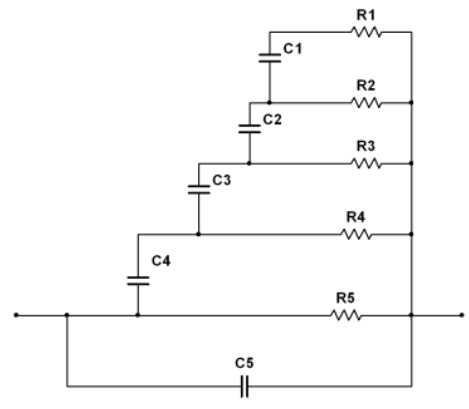

Figure 4. RC ladder network. For the $1 \mathrm{~m}$ cable of Figure. 2 with 32 sections, R $1=6.5199 \mathrm{e} 8 \Omega$, $2=4.5611 \mathrm{e} 6 \Omega, \mathrm{R} 3=7.0987 \mathrm{e} 6 \Omega, \mathrm{R} 4=1.3797 \mathrm{e} 7 \Omega$, $\mathrm{R} 5=4.2357 \mathrm{e} 9 \Omega, \mathrm{C} 1=0.54018 \mathrm{e}-15 \mathrm{~F}, \mathrm{C} 2=1.3475 \mathrm{e}-$ $15 \mathrm{~F}, \mathrm{C} 3=2.9342 \mathrm{e}-15 \mathrm{~F}, \mathrm{C} 4=1.9026 \mathrm{e}-15 \mathrm{~F}$, $\mathrm{C} 5=1.65 \mathrm{e}-12 \mathrm{~F}$. 


\subsection{Laplace SPICE element model for a two wire cable}

The admittance matrix of a network relates the current to the voltages at each of its ports [14]. A two wire cable of any length forms a two port network. For a two port network, the equation below shows the relationship between current and voltage at the input (port 1) and output (port 2).

$$
\left[\begin{array}{l}
I_{1} \\
I_{2}
\end{array}\right]=\left[\begin{array}{ll}
Y_{11} & Y_{12} \\
Y_{21} & Y_{22}
\end{array}\right]\left[\begin{array}{l}
V_{1} \\
V_{2}
\end{array}\right]
$$

Since the cable is a reciprocal network, $Y_{12}=Y_{21}$. The admittance parameters matrix of the cable can be measured using a vector network analyzer over a desired frequency range. This requires placing appropriate connectors between the cable and the network analyzer and impedance termination of $50 \Omega$ at the end of cable. The admittance parameters matrix of the two wire cable can also be obtained from a finite element analysis software. For a $1 \mathrm{~m}$ long cable with the cross-section shown in Figure 2, the admittance parameter matrix has been obtained from a finite element solver (ANSYS-HFSS) over a frequency range of $10 \mathrm{KHz}-100 \mathrm{MHz}$.

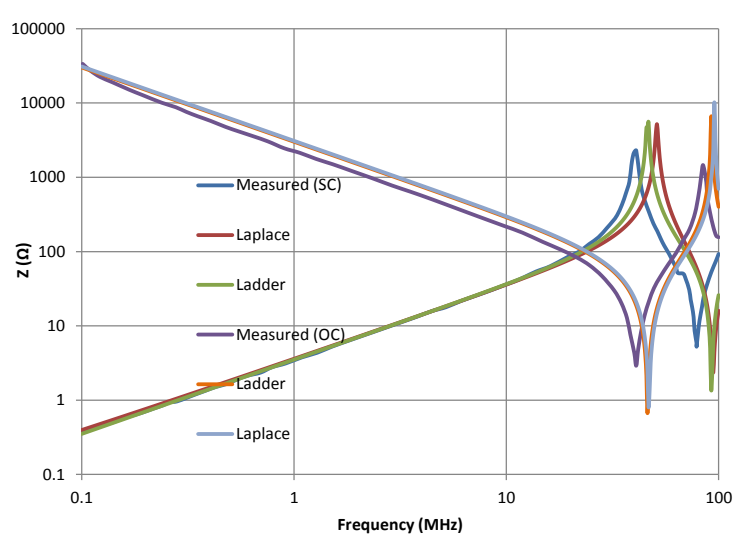

Figure 5. Input Impedance of $1 \mathrm{~m}$ Cable Terminated in Short Circuit (SC) and Open Circuit (OC)

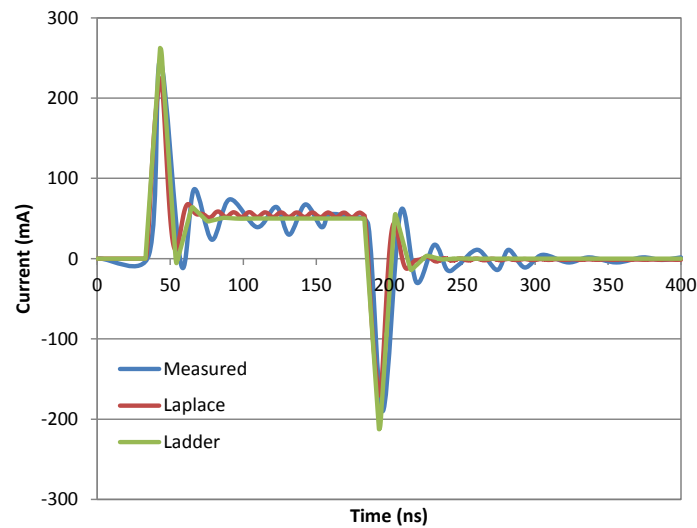

Figure 6. Transient Response of $1 \mathrm{~m}$ Cable Terminated in a $750 \Omega \mathrm{Load}$, Connected to $50 \Omega$ Source and an Input Pulse of 40V (Starts at $t=30$ ns with Rise Time of 10ns, Duration 150ns, Fall Time of 10ns)

The admittance parameters obtained are a function of frequency and therefore can be expressed as a function in the Laplace domain $(s=i * 2 \pi f)$. A rational function approximation of the admittance matrix $Y(s)$ is determined by using the vector fitting algorithm [9]. The admittance parameters, or in other words, the elements of the matrix $Y(s)$ of the network are expressed in pole residue form by this algorithm and are given as:

$$
Y_{m n}(s)=\sum_{i=1}^{N} \frac{R_{i, m n}}{\left(s-s_{i}\right)}+D_{m n}
$$

Where the N poles $s_{1}$ to $s_{N}$ are either real or complex conjugate pairs, their corresponding residues $R_{i}$ are either real or complex conjugate pairs, and $\mathrm{D}$ is real. The vector fitting algorithm expresses all the parameters in the admittance matrix with the same set of poles and different residues unless two of the parameters in the admittance matrix are equal.

For a circuit simulation, the rational function approximation should satisfy stability, causality, and passivity criteria. The vector fitting algorithm [9] creates an approximation that is stable and causal. The algorithm presented in [16] ensures passivity by checking whether the eigenvalues of $\operatorname{Re}[Y(s)]$ matrix are positive for all frequencies. If the criterion is not satisfied, it uses a perturbation technique to reformulate the rational function approximation.

A two port admittance matrix can be represented by an equivalent network in pi topology [14]. The admittance parameters form the branches in the pi topology network. Traditionally, standard network 
elements represent the admittance parameters. When rational function approximations of admittance parameters are in the Laplace domain, the branches of the pi topology can be replaced with these functions as shown in Figure 7. Each of the terms in the rational function approximation can be represented as Laplace elements in SPICE. By using a pi topology, there are no SPICE nodes inside the two ports of the cable. However, there are as many parallel Laplace SPICE elements between the nodes as the number of poles (corresponding to the terms in summation) used in approximating the admittance parameter. For example, the Laplace SPICE element of $Y_{m n}$ corresponding to, say, the first $(i=1)$ pole $s_{1}$ (assumed to be real) is given as:

GXXX m n LAPLACE $\{\mathrm{V}(\mathrm{m}, \mathrm{n})\}\left\{R_{1, m n} /\left(s-s_{1}\right)\right\}$

For a complex conjugate pole pair, say, $s_{2}$ and its conjugate, the Laplace SPICE element is given as:

GXXX m n LAPLACE $\{\mathrm{V}(\mathrm{m}, \mathrm{n})\}\left\{(A * s+E) /\left(s^{2}+B * s+C\right)\right\}$

where the values of $\mathrm{B}, \mathrm{C}, \mathrm{A}$, and $\mathrm{E}$ are:

$B=-2 * \operatorname{Re}\left(s_{2}\right)$

$C=\left|s_{2}\right|^{2}$

$A=-B$

$E=-2 * \operatorname{Re}\left(R_{2} s_{2}\right)$

The constant $D_{m n}$ is represented in SPICE as:

GXXX m n LAPLACE $\{\mathrm{V}(\mathrm{m}, \mathrm{n})\}\left\{D_{m n}\right\}$

All the poles of the rational function and $D_{m n}$ are expressed as $G$ elements (Voltage dependent current source) in the SPICE netlist. Similarly, all branches in the pi topology are expressed as G elements to create SPICE model of the cable. For the $1 \mathrm{~m}$-long two wire cable, a rational function approximation with as few as 20 poles is sufficient to model the cable. The computed and the measured [5] values of impedance when terminated in short and open circuits, as shown in Figure 2, are found to agree well. The time domain response of the cable to a $40 \mathrm{~V}$ pulse with rise and fall times of $10 \mathrm{~ns}$ is shown to agree well with measurements [5] and the ladder network representation.

The computation was repeated for a $10 \mathrm{~m}$ long cable of the same cross section. Both models were tested in time domain by applying a $40 \mathrm{~V}$ pulse of $10 \mathrm{~ns}$ rise time. The results obtained from the two models, as shown in Figure 8, agree well.

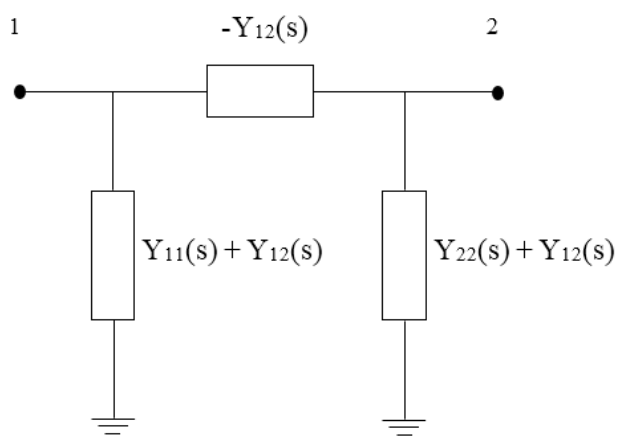

Figure 7. Two Port Network Model of two Wire Cable with Admittance Parameters in Laplace Domain

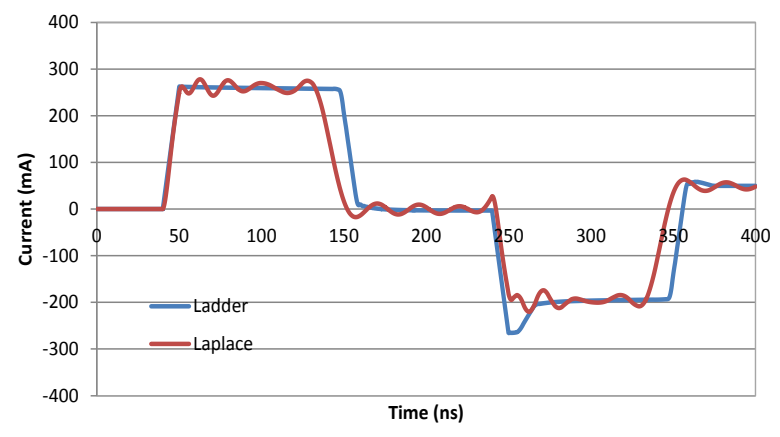

Figure 8. Transient Response of 10m Cable Terminated in a $750 \mathrm{Ohm}$ Load, Connected to $50 \mathrm{Ohm}$ Source and an Input Pulse of $40 \mathrm{~V}$ (Starts at $\mathrm{t}=40 \mathrm{~ns}$ with Rise Time of 10ns, Duration 190ns, Fall Time of 10ns)

\section{CONCLUSION}

Two SPICE models have been obtained to simulate a system that uses a long cable and switching pulses with short rise times. The LTspice simulator was used to simulate the system. From the simulation of a long cable $(1 \mathrm{~m}$ and $10 \mathrm{~m})$, it is observed that both models present solutions with good accuracy. The ladder network model is composed of several internal nodes in the cable, while the Laplace SPICE element model requires no internal nodes to represent the cable. The number of internal nodes increases with the length of the cable in the ladder network model. The SPICE circuit model netlist for the cable using Laplace elements 
has fewer lines. However, the computation times to perform a time domain simulation using the Laplace SPICE element model are longer than those of the ladder network model. This is due to the fact that while performing transient analysis in SPICE, the impulse response of the Laplace elements are calculated and convolved with input signal. If the model arrived at using Laplace elements is not passive, numerical inaccuracy results. If steps are taken to avoid this problem when synthesizing the model, accurate transient analysis can, however, be realized. Although either of the methods used to obtain a SPICE model for the cable is appropriate in terms of accuracy, the use of vector fitting algorithm to obtain a model is found to be elegant and likely to be more suitable for multiconductor cables.

\section{REFERENCES}

[1] Wei Z., "The Electromagnetic Interference Model Analysis of the Power Switching Devices", TELKOMNIKA Indonesian Journal of Electrical Engineering, Vol. 11, No. 1, pp.167-72, 2013.

[2] Clayton R. Paul, "Introduction to Electromagnetic Compatibility", John Wiley \& Sons, 2006.

[3] Gianluca Sena, Roberto Marani, Gennaro Gelao, Anna Gina Perri, "A Comparative Study of Power Semiconductor Devices for Industrial PWM Inverters", International Journal of Power Electronics and Drive System (IJPEDS),Vol. 7, No. 4, pp. 1420-1428, December 2016.

[4] N. Idir and Y. Weens and M. Moreau and J. J. Franchaud, "High-Frequency Behavior Models of AC Motors", IEEE Tran. On Magnetics, Vol: 45, No.1, pp. 133 - 13, Jan. 2009.

[5] Y.Weens, N. Idir, R. Bauiere, and J. J. Franchaud, "Modeling and simulation of unshielded and shielded energy cables in Frequency and Time domains", IEEE Trans. On Magnetics, Vol. 42, No. 7, pp. 1876-1882, December 2016.

[6] B. Gustavsen and A. Semlyen, "Rational approximation of frequency domain response by Vector Fitting", IEEE Trans. Power Delivery, Vol. 14, No. 3, pp. 1052-106, , July 1999.

[7] B. Gustavsen, "Improving the pole relocating properties of vector fitting", IEEE Trans. Power Delivery, Vol. 21, No. 3, pp. 1587-1592, July 2006.

[8] D. Deschrijver, M. Mrozowski, T. Dhaene, and D. De Zutter, "Macromodeling of Multiport systems using a Fast implementation of Vector Fitting Method", IEEE Microwave and Wireless Components Letters, Vol. 18, No. 6, pp.383-385, June 2008.

[9] The Vector fitting website - https://www.sintef.no/projectweb/vectfit/ May 15th 2017.

[10] Ivica Stevanovic, Bernard Wunsch, Gian Luigi Madonna, Stanislav Skibin, "High-Frequency Behavioral Multiconductor Cable Modeling for EMI Simulations in Power Electronics", IEEE Trans. On Industrial Informatics, Vol. 10, No. 2, pp. 1392-1400, May 2014.

[11] Eli Steenput, "A Spice circuit can be synthesized with a specified set of S-parameters", Vrije Universiteit Brussel, pp 1-12.

[12] Antonije R. Djordjevic, "SPICE-Compatible Models for Multiconductor Transmission Lines in Laplace-Transform Domain”, IEEE Trans. on Microwave Theory and Techniques, Vol. 45, No. 5, pp. 569-579, May 1997.

[13] Yemna Bensalem and Mohamed Naceur Abdelkrim, "Modeling and Simulation of Induction Motor based on Finite Element Analysis", International Journal of Power Electronics and Drive System (IJPEDS), Vol. 7, No. 4, pp. 1100-1109, December 2016.

[14] David M. Pozar, “Microwave Engineering”, John Wiley \& Sons, 1998.

[15] Sarhan M. Musa and Matthew N. O. Sadiku, "Finite Element Analysis for Five Transmission Lines in Multilayer Dielectric Media", International Journal of Advances in Applied Sciences (IJAAS), Vol. 1, No. 4, pp. 181-190, December 2012.

[16] B. Gustavsen and A. Semlyen, "Enforcing passivity for Admittance Matrices approximated by rational functions", IEEE Trans. Power systems, Vol. 16, No. 1, pp.97-104, Jan 2001. 